\title{
Estepa y rutas de la seda frente al espejo de la Región Autónoma Uigur de Xinjiang. Fronteras fluidas y construcción de narrativas histórico-identitarias
}

\author{
Steppe and Silk Routes in front of the mirror of Xinjiang's Autonomous \\ Region of Uyghur. Fluid borders and construction \\ of historical-identity narratives
}

\section{Chiara Olivieri}

Universidad de Granada. olivieric@ugr.es

Recibido: 10/04/2018. Aceptado: 04/08/2018

Resumen: La propuesta para este capítulo es la de analizar cómo, a partir de las fronteras imperiales establecidas bajo el período del Gobierno Qing, las poblaciones "bárbaras"/fronterizas han sido incluidas -más o menos forzosamente- en una definición -más o menos amplia- de "China". Tras una sucinta introducción histórica sobre el territorio, mi objetivo es el de empezar a hacer hincapié en la influencia, a nivel cultural, lingüístico, identitario y religioso, de la región que -tras su anexión al Imperio Qing-ha pasado a denominarse Xinjiang, en relación con los actores políticos y sociales circunstantes, y tratando de puntualizar unas especificidades históricas de la región, en un contexto eurasiático más amplio.

Palabras clave: Uigur; Xinjiang; identidad; narrativas históricas; colonialismo.

\begin{abstract}
The proposal for this chapter is to analyze how, from the imperial borders established under the Qing government period, the "barbarian"/border populations have been included -more or less forcedly, -in a -more or less broad-definition of "China." After a succinct historical introduction about the territory, my goal is to begin to emphasize the cultural, linguistic, identity and religious influence of the region that -after its annexation to the Qing Empire -has been renamed Xinjiang, in relation to the surrounding political and social actors, and trying to point out some historical specificities of the region, in a wider Eurasian context.
\end{abstract}

Keywords: Uyghur; Xinjiang; identity; historical narratives; colonialism.

\) Olivieri, Chiara. 2018. "Estepa y rutas de la seda frente al espejo de la Región Autónoma Uigur de Xinjiang. Fronteras fluidas y construcción de narrativas histórico-identitarias". Quaderns de Filologia: Estudis Literaris XXIII: 23-38. doi: 10.7203 /qdfed.23.13428 



\section{Introducción}

La propuesta para este artículo es la de analizar cómo, a partir de las fronteras imperiales establecidas bajo el período de gobierno Qing, las poblaciones fronterizas han sido incluidas de una forma más o menos forzosa en una definición de "China". Mi objetivo es el de empezar a hacer hincapié en la influencia, a nivel cultural, identitario y religioso, de la región que -tras su anexión al Imperio Qing- ha pasado a denominarse Xinjiang ${ }^{1}$, en relación con los actores políticos y sociales circundantes. Unas narrativas históricas han sido producidas y perpetradas por parte de un actor externo, con el claro intento de construir un escenario instrumental para establecer un control -político, económico, social, cultural e identitario- sobre la región. El proceso de construcción de una identidad territorial en XUAR es el resultado de siglos de convivencia y contactos no solo comerciales -como en muchos casos la literatura ha querido subrayar-, sino religiosos, culturales y lingüísticos. A partir de la conquista Qing, hasta la fundación de la República Popular de China (RPCh), se tratará de analizar las narrativas de construcción identitaria impuestas en la región, así como de evidenciar sus causas y consecuencias políticas y económicas en la actualidad de la región, en un contexto eurasiático más amplio. La "creación" de XUAR se enmarcará pues en un más amplio escenario de construcción estatal/comunitaria en sentido andersoniano a saber, un lugar que, "independientemente de la desigualdad y la explotación que en efecto puedan prevalecer en cada caso, ... se concibe siempre como un compañerismo profundo, horizontal" (Anderson, 1993: 25). Así pues, el objetivo es centralizar lo que históricamente se ha construido como periferia, subrayando su valor no solamente geoestratégico y económico, sino como pieza de conexión indispensable entre China y Asia Central. Se tratará por tanto de desenmascarar un plan político/económico de control territorial,

\footnotetext{
${ }^{1}$ Desde el principio, consideramos necesaria una precisión terminológica: nos referiremos a la Región Autónoma Uigur de Xinjiang con la abreviación XUAR - Xinjiang Uyghur Autonomous Region- cuando hablemos del territorio geopolítico tal y como lo establece y conforma la República Popular de China (RPCh): haremos referencia a XUAR cuando nombremos cuestiones relacionadas con las políticas impuestas por el Estado Central sobre ese territorio - esto es, cuando detallemos situaciones que implican una relación entre la región y la RPCh-. Elegimos utilizar una abreviación, en vez de usar el término Xinjiang, para remarcar y hacer patente la oficial -y no real- "autonomía" de la región, así como el reconocimiento de su "pertenencia" al pueblo Uigur.
} 
instrumental para potenciar beneficios comerciales y financieros, y las consecuencias socio-políticas implicadas. En suma, se propone aquí reflexionar acerca de cómo unas narrativas inclusivas e igualitarias esconden un plan de explotación extractivista -a nivel natural y humano- en XUAR, y legitiman políticas de control que a su vez conllevan prácticas de violencia - ontológica y física- $\mathrm{y}$ desigualdades.

\section{El valor de las estepas}

Punto de partida de esta discusión es cuestionar desde el principio las narrativas acerca de lo que comúnmente se define "Ruta de la Seda", desmontando el imaginario exotizante sugerido por su ideador, el geógrafo alemán -concentrado, en sus investigaciones en China desde 1868 hasta 1872, en analizar la posibilidad de establecer una colonia económica alemana en China en busca de energía, industria, carbón, acero y ferrocarriles- Ferdinand von Richtofen en 1877 (Millward, 2018b); concentrando, esto es, el foco de la atención no en una ruta que conectaba en línea recta a Roma con China, y en la que mercaderes independientes transportaban mercancías de lujo -especias, manufacturas, gemas y, cómo no, seda- a través de una serie de enclaves -las ciudades de la Ruta de la Seda-directamente de un extremo a otro del recorrido. Como bien apunta Christian (2000: 68-69), en primer lugar, es importante subrayar la pluralidad de las rutas, pues estas consistían en una red en continua transformación de caminos, en los que se concentraban diferentes tipos de intercambios, constituyéndose pues como los recorridos por los que se intercambiaban bienes, ideas y personas entre los mayores regiones de Afro-Eurasia.

Eurasia Central - conocida también como Asia Central, Asia Interior, Eurasia Interior, etc.- es una vasta región que ocupa el corazón del continente eurasiático, y que históricamente ha tenido relaciones fundamentalmente pacíficas con las mayores civilizaciones de China, India, Persia, el Mediterráneo y Europa. Pese a su gran diversidad geográfica, sus estepas y desiertos han propiciado un sistema económico - esencialmente pastoreo nómada- que produjo una serie de consecuencias primordiales a nivel militar, y que es preciso subrayar: la cría y el dominio de caballos propició que los estados semi-nómadas de Asia Central tuviesen una importante ventaja sobre los sedentarios, desde el primer milenio A.d.N.E. hasta tiempos relativamente recientes. Asi- 
mismo, las poblaciones de la región han sido conductoras de bienes, religiones, tecnologías, artes, ideas, ADN a través de los bordes de las regiones eurasiáticas. Estas se caracterizan por poseer una enorme diversidad ecológica y geográfica, lo que evidentemente favorece que los intercambios de productos -materiales e inmateriales- entre ellas se intensifiquen y diversifiquen (Christian, 2000; Pulleyblank, 2002; Sherratt, 2006; Millward, 2013).

En este panorama de rutas y conexiones, es preciso hacer patente la estrecha interconexión que deriva en el mismo establecimiento y progreso de las civilizaciones implicadas; como afirma Andrew Sherratt (2006: 31):

Just as human personality emerges only through social interaction, without which it atrophies, so human cultures develop their potential only in a larger context than that of a single society.

Y efectivamente, se ve necesario romper con la imagen tradicional según la cual diferentes civilizaciones -ajenas e independientes las unas de las otras- utilizan las Rutas de la Seda con fines meramente económicos, comerciales y bélicos, y fomentar en cambio una visión por la que el carácter de todas ellas fue plasmándose a raíz de esos contactos. Asimismo, los propios intercambios de técnicas, productos y medios de producción se vieron afectado por el diálogo -social, político, diplomático y, cómo no, comercial- establecido a lo largo de los vastos territorios esteparios de Asia Central. De entre ellos, la región que hoy en día conocemos como XUAR se impone, por su posición geoestratégica, así como por su historia y sus características ecológicas, como un eje central para que estas conexiones fluidas se realizaran entre diferentes actores: la gestación y el desarrollo de nacionalismos, religiones, tecnologías, así como el papel que desempeña como filtro de culturas hacen de este territorio una pieza fundamental para comprender la fluidez de las relaciones no sólo ya en épocas antiguas, sino en los geo-esquemas $^{2}$ (Millward, 2018b) actuales, remarcando la necesidad de analizar un eje norte-sur en las conexiones -además del más explotado a nivel

\footnotetext{
${ }^{2}$ Una entidad conceptual metageográfica a escala regional con dimensiones territoriales, históricas, políticas, culturales, económicas, legales, estratégicas, etc., susceptible de crear manipulaciones con propósitos geopolíticos e ideológicos (Millward, 2018b).
} 
académico, investigador y literario eje este-oeste ${ }^{3}-\mathrm{y}$ redignificar el papel de las poblaciones túrquicas (Golden, 2006) indígenas -nómadas y seminómadas-como elemento trans-ecológico y trans-civilizacional de intermediario en estos intercambios (Christian, 2000).

\subsection{Mapeando las Rutas de la Seda}

La fluidez de los intercambios y de las fronteras se ve sometida, a partir del siglo XVII (Perdue, 1998) a procesos de fijación estratégica por parte de los tres poderes que se contendían el control de la zona: el imperio ruso, la dinastía Qing, y el imperio mongol de Zungaria -aunque este último cayó bajo la presión del ejército Manchú a mediados del siglo XVIII. Antes de esa fecha, el establecimiento de fronteras fijadas para sus dominios no había constituido una de las mayores preocupaciones para los gobernantes de los estados europeos y asiáticos (Perdue, 1998: 264); sin embargo, su necesidad de control territorial -y de recursos-, así como la voluntad de ratificar los resultados de los enfrentamientos armados, promovieron la producción y difusión de mapas y estudios de notable precisión, por parte de los tres actores, cuyos intereses quedaron fijados en fronteras "colonialmente determinadas" (Anderson, 1993: 239); evidentemente, el valor instrumental de los mapas es un aspecto a subrayar, en la creación de narrativas y la imposición de poder sobre un territorio. Los intercambios mutuos entre las tres potencias mencionadas, sus confrontaciones militares y sus acciones diplomáticas, en definitiva, establecieron las fronteras del y en el territorio de Asia Central, en la mayor parte de los casos anexionando y/o separando tribus, familias, pueblos sometidos a gobiernos alternados por parte de una potencia u otra, mediante medidas de "clasificación totalizadora" (Anderson, 1993: 240). Así pues, los movimientos poblacionales se someten a la discreción de los gobernantes, que no sólo pueden aprobarlos o prohibirlos, sino que ganan una herramienta de control sobre sus sujetos, clasificándolos y definiéndolos étnicamente (Hostetler, 2001; Brophy, 2016; Leibold, 2007). A ello se suma, cómo no, un claro establecimiento de jerarquías de poder en la gestión de las estructuras y me-

\footnotetext{
${ }^{3}$ Eje que, según afirma Andrew Sherratt (2006), incidió en la formación de la idea dicotómica de Oriente vs. Occidente a raíz y como resultado de los hallazgos arqueológicos, que contrapondrían las dos "civilizaciones" en base a sus técnicas metalúrgicas (fundido/forjado).
} 
dios de producción indígenas; poblaciones tradicionalmente nómadas, con sus sistemas de manejo de recursos, se ven coercidas y obligadas a adaptarse a las políticas dictadas por el estado, que ejerce un control, por tanto, no sólo ya territorial, sino humano.

Los mapas, pues, se configuran como una herramienta fundacional de las narrativas de pertenencia/anexión identitaria que forman parte de las estrategias estatales de control y sumisión, creando una imagen de unidad/homogeneidad, instrumental para mantener unas jerarquías de poder impuestas por los gobernantes, "prefiguran[do] los nacionalismos oficiales" (Anderson, 1993: 243).

\section{Narrativas identitarias. La construcción de lo "chino"}

Desde que los amplios territorios de Altishahr-conocido también como Turkestán Oriental, incluye la cuenca de Tarim y los oasis musulmanes circundantes- y Zungaria - la cuenca triangular al norte de la cordillera de Tianshan, definida por las montañas de Altai en el este y el valle del río Ilí y el lago Balkash en el oeste- (Millward, 1999: 61) fueron incluidos en los mapas del territorio perteneciente a la dinastía ( $r u$ bantu $)^{4}$ :

...not only did the conquest of these expanses from the Zunghars literally put Xinjiang on the Qing map, but mapping and research into their historical geography were instrumental in making them part of a new, expanded conception of China (Millward, 1999: 62).

Los "mapas históricos" (Anderson, 1993), pues, a partir de la época Qing ${ }^{5}$, sirvieron como una poderosa herramienta para que la ocupación

\footnotetext{
4 "The classical term ru bantu is here perhaps best translated less literally, as "become part of our territory," for the word was commonly used in Qing times to describe the incorporation into the Qing empire" (Millward, 1999: 61).

${ }^{5}$ Se hace, a lo largo de este estudio, especial hincapié en la última etapa dinástica de China, coincidiendo con autores de la corriente historiográfica de la denominada New Qing History (Crossley, 1999; Elliott, 2001; Hostetler, 2001; Millward, 1998; Perdue, 2005; Rawski, 2015); se considera este un momento cabal en el establecimiento de las fronteras nacionales del país; coincidiendo, cronológicamente, con el surgimiento de los nacionalismos en Europa -o los que Benedict Anderson denomina "nacionalismos occidentales", cfr. Anderson, Benedict. 2001. "Western nationalism and eastern nationalism: Is there a difference that matters?" New Left Review 21: 31-42-, refleja el modelo de construcción estatal nacionalista cuyas consecuencias ontológicas y epistemológicas homogeneizantes se pretende evidenciar aquí. Para obras más generales
} 
del territorio que hoy conocemos como XUAR se fundiera con la construcción de narrativas de pertenencia histórica del territorio a "China": a través del establecimiento de bases militares -como estrategia de "pacificación" (Clarke, 2003)- en la región, la migración masiva de mercaderes Han -con el pretexto de fomentar y controlar el mercado intra-asiático y "modernizar" el territorio infraexplotado por las poblaciones indígenas (Bovingdon, 2010; Cliff, 2017)- y el fomento de órganos gubernamentales y económicos, la dinastía Manchú consiguió una serie de beneficios a nivel político y social: por un lado, la importancia geoestratégica de la región facilitaba las conexiones comerciales con otras zonas de Asia Central (Millward, 2007; Brophy, 2016); asimismo, la vastedad territorial proporcionaba una adecuada almohadilla de seguridad para el surplus de población.

La guetización y separación de los diferentes grupos poblacionales en la región -indígenas, Han y Manchúes-, ratificadas incluso por decretos imperiales, no tardaron en crear una serie de separaciones ontológicas, jerárquicas e incluso físicas entre la población que, supuestamente, convivía. La élite mercantil y, paulatinamente, gubernamental de Xinjiang fue pasando de manos Manchúes a manos Han, así como el sistema de tasación era más ligero hacia los indígenas, para no crear malestares (Millward, 2007; Brophy, 2016).

Durante los tumultuosos años de transición del gobierno de la dinastía Qing a la República, los procesos de construcción estatal han conllevado el establecimiento de narrativas de alteridad identitaria, imprescindibles para mantener el control sobre la región. Los pueblos "no sínicos" (Leibold, 2007) han sido objeto de definiciones alternadas por parte de los sucesivos órganos de poder, dependiendo de intereses políticos, económicos y geoestratégicos: desde una inicial postura antino-sínicos -instrumental para invalidar la autoridad del poder Qing/ Manchú a los ojos del pueblo- se deriva hacia discursos unitarios y homogeneizadores, en el momento en que la mayoría se ve en la necesidad de ser respaldada por sus "Otros internos" frente a las amenazas de los "Otros externos" -léase, las potencias coloniales-, y justificar su soberanía sobre la totalidad de los territorios colonizados y anexiona-

acerca del concepto de nacionalismo, cfr. también Hobsbawm, Eric. 2000. Naciones y Nacionalismo desde 1780. Barcelona: Crítica; Mukherji, Partha Nath. 2010. "Civic-secular and ethnic nationalisms as bases of the nation-state: multiculturalism at the crossroads?". Asian Ethnicities 11(1): 1-23. 
dos durante la época Qing. La teoría del darwinismo social, el mismo concepto biológico de raza (Dikötter, 2002) y la creciente influencia de sentimientos nacionalistas -que no han de considerarse meras "adquisiciones" derivadas de los contactos coloniales, sino más bien como una de las naturales consecuencias de la inclusión de China en nuestro Sistema-Mundo Moderno/Colonial han contribuido al establecimiento de narrativas identitarias que auxiliaran en la conformación de China como Estado-Nación moderno y preparado para su inclusión en un panorama internacional amplio y regido por reglas estancas (Leibold, 2007: 46).

La periferia, pues, se vuelve imprescindible para la realización de un diseño nacional/nacionalista, así como para proporcionar ingresos y recursos en la economía del país ${ }^{6}$. Sin embargo, el establecimiento de soberanía sobre territorios "útiles" conlleva necesariamente la "aceptación/inclusión/asimilación" de sus poblaciones indígenas, consideradas "inferiores" - numérica y culturalmente-. "China", por lo tanto, emerge como un actor diseñado ad hoc, aprovechando discursos inclusionistas y estáticos que, tanto desde dentro como desde fuera, se habían ido construyendo en torno a ella.

\section{Propagandas contemporáneas}

Si el punto focal que se quiere aquí evidenciar, pues, es la construcción de las Rutas de la Seda como una idea directamente conectada con procesos y tiempos de imperialismos e industrialización (Millward, 2018b), es preciso demorarse en analizar cómo todo esto se materializa, aún hoy en día, a nivel político, económico y social.

El relativamente reciente resurgimiento de la idea de unas rutas comerciales que conecten a China con el resto del planeta, a través de enclaves distribuidos a lo largo y a lo ancho de todo el territorio de Asia, Europa, África e incluso América Latina hace que se vuelva a abrir el debate acerca de algunas de las cuestiones que hasta ahora hemos tratado de evidenciar: la inclusión de territorios pertenecientes a las antiguas Rutas de la Seda en una definición amplia de lo que es China conlleva la

\footnotetext{
${ }^{6}$ Para datos concretos sobre la estrategia económica regional, y su impacto en el debate que nos corresponde sobre la estrategia colonial de China en la región de XUAR, Cfr. Sautman, 2000.
} 
patrimonialización de estos $-\mathrm{y}$ de sus recursos, paisajes y gentes- $\mathrm{y}$ la necesidad de mantenerlos bajo el control estatal.

El presidente de la RPCh Xi Jinping anunció en 2013, en dos discursos que realizó en Indonesia y Kazajistán, la apertura de las rutas comerciales eurasiáticas conocidas bajo la denominación de Belt and Road Initiative 7 (BRI), como estrategia para mejorar su competitividad internacional en un escenario de economía globalizada a nivel de producción, comercio, capital e internacionalización de recursos.

El proyecto económico -con evidentes consecuencias geoestratégicas-, partiendo del desarrollo de las infraestructuras de transporte y logística, presenta una estrategia que apunta a promover el papel de China en las relaciones globales, favoreciendo flujos de inversiones internacionales y salidas comerciales para los productos chinos, a través de una serie de rutas terrestres - que beben de los escenarios legendarios del comercio centrasiático de la China Han-y marítimas -forjadas en los relatos alrededor de la figura de Zheng He (Millward, 2018b)-, tratando de restablecer las antiguas vías de las Rutas de la Seda, y promocionando la creación de nuevos caminos, con el fin de conectar un más amplio número de territorios y países -alrededor de sesenta.

Sin querer analizar aquí con detenimiento los aspectos exquisitamente económicos y comerciales de la iniciativa, nuestro objetivo es más bien tratar de demostrar cómo los matices apuntados en los apartados anteriores construyen los discursos subyacentes a ella, cómo estos discursos reavivan algunas de las narrativas tradicionales de las Rutas de la Seda, y cómo estas medidas impactan a nivel social -epistemológico y ontológico- en la vida diaria de las poblaciones que habitan el territorio de XUAR; esto es, mirar a la BRI como un "geoesquema", tal y como lo define Millward (2018b): "una entidad conceptual metageográfica de escala regional con dimensiones territoriales, históricas, políticas, culturales, económicas, legales, estratégicas y otras susceptibles a la manipulación creativa con fines geopolíticos e ideológicos". Los estudios que cuestionan la naturaleza colonial de las relaciones que se establecen entre la RPCh y XUAR han proliferado en las últimas

\footnotetext{
7 También conocida como One Belt One Road Initiative (OBOR), The Belt \& Road (B\&R), Yi dai yi lu en chino. Aquí se utilizará la abreviación BRI -Belt and Road Initiative. La denominación en español "Iniciativa del Cinturón y Ruta de la Seda" se sustituye en ocasiones con la abreviatura NRS (Nueva Ruta de la Seda), para atestiguar su voluntad de continuidad con las Rutas de la Seda antiguas/tradicionales.
} 
décadas (Béller-Hann, 2008; Bovingdon, 2010; Gladney, 1991, 2004; Jacobs, 2016; Millward, 2018a, 2018b); asimismo, en tiempos muy recientes se están empezando a cuestionar las medidas de "desarrollo" y "modernización" de las infraestructuras promovidas por el proyecto; esto es, los procesos de gentrificación de los territorios involucrados, y el paradero de los beneficios reales de la iniciativa.

Si bien el presidente Xi hace especial hincapié en los efectos benéficos del proyecto, subrayando los principios de paz y cooperación, apertura e inclusión en los que se basa (Clarke, 2017), no hay que descuidar las políticas reales que se aplican en la región que nos corresponde, donde la implantación de la iniciativa coincide con una cada vez más rígida aplicación de medidas de control político, ideológico y social (Béller-Hann, 2008; Castets, 2015; Leibold \& Grose, 2016; Clarke, 2017; Millward, 2018a). Pese a los indudables beneficios económicos regionales -la tasa de crecimiento del PIB de XUAR ha superado la media nacional desde 2003 (Clarke, 2017: 4)-, las estrategias asimilacionistas que los acompañan - esto es, la implementación de procesos de sinización, léase hanización de la población, a través de medidas de represión de marcas identitarias autóctonas, como las lingüísticas, religiosas, tradicionales, etc.- son igualmente evidentes: se materializan a través de políticas migratorias -incentivos económicos para que ciudadanos Han se establezcan en el territorio- económicas -exclusión de parte de la población de ciertos ámbitos laborales-, sociales -guetización, separación- y policiales -control y represión de manifestaciones del pueblo Uigur (Clarke, 2017: 4). Beijing legitima su política, a nivel diplomático y a los ojos de la comunidad internacional, bajo el estandarte de la salvaguarda del "patrimonio" cultural que las Rutas de la Seda representan, a nivel global; asimismo, un mayor desarrollo económico del legendario enclave de conexión trans-euroasiática representado por XUAR favorecería la mitigación de las reivindicaciones autonomistas e independentistas de parte de su población; para mantener el escenario de "paz y armonía" y de beneficios globales pincelado por el Presidente Xi (Clarke, 2017: 1), el estado de seguridad se mantiene bajo la justificación de "lucha al terrorismo", acechando a la radicalización religiosa de la población los incidentes - en la mayoría de los casos, de naturaleza política y económica- que se verifican en la zona, y con el claro objetivo de poder mantener un control firme sobre el territorio, a través de la opresión de su pueblo. 


\section{Reflexiones finales}

El lugar geoestratégico que ocupa la actual región de XUAR ha favorecido, a lo largo de los siglos, el establecimiento de lazos comerciales, diplomáticos y coloniales de la zona, por parte de diferentes actores. Desde luego, el papel desarrollado por China, en sus diferentes etapas estatales, ha destacado como el más relevante, a largo plazo, y viendo la hodierna conformación socio-política del territorio -sin querer por ello obviar el papel de la comunidad internacional en la perpetración de -o en la falta de sanciones por- las medidas que a lo largo de este estudio se ha tratado de patentar.

Enclave fundamental de las antiguas vías de intercambios entre estados europeos y asiáticos, lo que hoy conocemos como XUAR ha sido cuna y receptáculo de productos - materiales, ideológicos, religiosos, humanos y genéticos- que a través y desde allá se han movido en dirección este-oeste y norte-sur, tras procesos de reelaboración y metabolización por parte de las poblaciones indígenas. De igual manera, su conformación topográfica y ecológica, así como sus recursos naturales han representado un fértil incentivo para el establecimiento de relaciones de poder en la zona, entre actores internos y externos. $\mathrm{Su}$ inclusión en lo que se ha definido como "China", su anexión definitiva a la RPCh, y el consiguiente asentamiento de órganos de gobierno y comunidades Han, mas especialmente las políticas de "modernización" por ellos promulgadas, promovidas e impuestas representan uno de los factores que aquí nos hemos propuesto subrayar: los discursos de inclusión de XUAR en un más amplio proyecto de establecimiento -o, como se ha sugerido, de restablecimiento- de conexiones trans-eurasiáticas, de intercambios y desarrollo económico supranacional esconden, más o menos veladamente, la necesidad de la RPCh de sedar las aspiraciones independentistas/autonomistas de parte de la población Uigur. Las cifras publicadas, relativamente al crecimiento económico regional promocionado por las medidas estatales confirman el superficial beneficio, en términos monetarios e infraestructurales, de las políticas de la RPCh; tras la disolución de la URSS y el surgimiento de los Estados de Asia Central independientes, se crearon muchas nuevas oportunidades para el desarrollo de XUAR no sólo como parte de China, sino también para que éste desempeñara un papel activo en el establecimiento de relaciones más estrechas entre la propia RPCh y los países de Asia Central. 
Desde el "Plan de Desarrollo del Oeste", lanzado en 2001, el gobierno de la RPCh ha tratado de aprovechar la localización de la región para empujarse de manera cada vez más contundente hacia el oeste esto es, Asia Central y Europa-, y reafirmar su posición global.

Explotar las narrativas ideológicas, literarias y fantásticas que, a lo largo de los últimos siglos, se han construido alrededor de las Rutas de la Seda para promover un proyecto económico y su propia imposición en un panorama comercial y monetario global y globalizado no debería ser suficiente para invisibilizar las consecuencias sociales prácticas de dicho proyecto: industrializar, modernizar, desarrollar una región, pese a los beneficios económicos incontestables que conlleva, trae consigo "necesarios" reajustes poblacionales y ontológicos que no pueden ser obviados. Homogeneización de las gentes, desplazamientos forzosos, gentrificación, absorción cultural, epistemicidios, represiones étnicas, lingüísticas, religiosas e identitarias, explotación y extracción desigual de recursos, exclusión, segregación, violencia; son factores a desenmascarar y visibilizar, denunciar y atacar, a la hora de hacer un balance de la situación actual, y del papel de la RPCh en la geopolítica mundial.

\section{Bibliografía}

Anderson, Benedict. 1993. Comunidades Imaginadas. Reflexiones sobre el origen y la difusión del nacionalismo. México: Fondo de Cultura Económica.

Christian, David. 2000. Silk Roads o Steppe Roads? The Silk Roads in World History. En Christian, David \& Benjamin, Craig (ed.) Realms of the Silk Roads: Ancient and Modern. Silk Road Studies IV. Turnhaut: Brepols.

Bellér-Hann, Ildikó; Cesàro, M. Cristina; Harris, Rachel \& Smith Finley, Joanne (ed.). 2007. Situating the Uyghurs Between China and Central Asia. Aldershot: Ashgate.

Beller-Hann, Ildikó. 2008. Community Matters in Xinjiang 1880-1949. Towards a Historical Anthropology of the Uyghur 17. Leiden: Brill.

Bovingdon, Gardner. 2010. The Uyghurs: Strangers in their own Land. New York: Columbia University Press.

\footnotetext{
${ }^{8}$ A partir de 2001, el gobierno chino ha ofrecido políticas preferenciales a las regiones occidentales -Gansu, Guizhou, Ningxia, Qinghai, Shaanxi, Sichuan, Tibet, Xinjiang, Yunnan, y el municipio de Chongqing- en términos de aportación de capital, apertura a las inversiones internas y externas, desarrollo de la ciencia, la educación, los recursos humanos y las infraestructuras.
} 
Brophy, David. 2016. Uyghur Nation: Reform and Revolution on the Russia-China Frontier. Cambridge, MA: Harvard University Press.

Castets, Rémi. 2015. The modern Chinese state and strategies of control over Uyghur Islam. Central Asian Affairs 2(3): 221-245.

Clarke, Michael. 2003. Xinjiang and China's Relations with Central Asia, 1991-2001: Across the 'Domestic-Foreign Frontier'? Asian Ethnicity 4(2): 207-224.

Clarke, Michael. 2017 (20 de julio). The Belt and Road Initiative and China’s Xinjiang Dilemma: "Connectivity" Versus Control? The Central Asia-Caucasus ANALYST. https:/www.cacianalyst.org/publications/ analytical-articles/item/13458-the-belt-and-road-initiative-and-china's-xinjiang-dilemma-"connectivity"-versus-control?.html.

Cliff, Tom. 2017. Oil and Water: Being Han in Xinjiang. Chicago: University of Chicago Press.

Crossley, Pamela K. 1999. A Translucent Mirror: History and Identity in Qing Imperial Ideology. Berkeley: University of California Press.

Dikötter, Frank. 2002. Race in China. En Goldberg, David T. \& Solomos, John (ed.) A Companion to Racial and Ethnic Studies. Oxford: Blackwell, 495-510.

Elliot, Mark C. 2001. The Manchu Way: The Eight Banners and Ethnic Identity in Late Imperial China. Stanford: Stanford University Press.

Fairbank, John King (ed.). The Chinese World Order: Traditional China's Foreign Relations. Cambridge, MA: Harvard University Press.

Fairbank, John King \& Goldman, M. (2006). China: A New History. Cambridge, MA: Harvard University Press.

Fletcher, Joseph. F. 1968. China and Central Asia, 1368-1884. En Fairbank, John King (ed.) The Chinese World Order: Traditional China's Foreign Relations. Cambridge, MA: Harvard University Press, 206-224.

Gladney, Dru. 1991. Muslim Chinese: Ethnic Nationalism in the People's Republic. Cambridge, MA: Harvard University Press.

Gladney, Dru. 1998. Ethnic Identity in China: The Making of a Muslim Minority Nationality. Wadsworth: Harcourt Brace College Publishers.

Gladney, Dru. 2004. Dislocating China: Muslims, Minorities, and Other Subaltern Subjects. Chicago: University of Chicago.

Golden, Peter. 2006. Some thoughts on the origins of the Turks and the shaping of the Turkic peoples. En Mair, Victor (ed.) Contact and Exchange in the Ancient World. Honolulu: University of Hawai'i Press, 136-157.

Hostetler, Laura. 2001. Qing Colonial Enterprise: Ethnography and Cartography in Early Modern China. Chicago / Londres: University of Chicago Press.

Jacobs, Justin. 2016. Xinjiang and the Modern Chinese State. Seattle / Londres: University of Washington Press. 
Karrar, Hasan Haider. 2009. The New Silk Road Diplomacy: China's Central Asian Foreign Policy since the Cold War. Vancouver / Toronto: UBC Press.

Leibold, James. 2007. Reconfiguring Chinese Nationalism: how the Qing frontier and its indigenes became Chinese. New York: Palgrave Macmillan. Leibold, James \& Grose, Timothy. 2016. Islamic Veiling in Xinjiang: The Political and Societal Struggle to Define Uyghur Female Adornment. The China Journal 76: 78-102.

Mackerras, Colin. 1973. The Uighur Empire according to the T'ang dynastic histories: a study in Sino-Uighur relations, 744-840. Columbia: University of South Carolina Press.

Mair, Victor. (ed.). 2014. Reconfiguring the Silk Road: New Research on EastWest Exchange in Antiquity: the papers of a symposium held at the University of Pennsylvania Museum of Archaeology and Anthropology, March 19, 2011. Philadelphia: University of Pennsylvania Museum of Archaeology and Anthropology.

Mallory, James Patrick \& Mair, Victror. 2008. The Tarim Mummies: Ancient China and the Mystery of the Earliest Peoples from the West. London: Thames \& Hudson.

Millward, James A. 1998. Beyond the Pass. Economy, Ethnicity, and Empire in Qing Central Asia, 1759-1864. Stanford: Stanford University Press.

Millward, James A. 1999. "Coming onto the Map": "Western Regions" Geography and Cartographic Nomenclature in the Making of Chinese Empire in Xinjiang. Late Imperial China 20(2): 61-98.

Millward, James A. 2002. Qing Colonial Enterprise: Ethnography and Cartography in Early Modern China (review). Journal of Interdisciplinary History 33(2): 347-348.

Millward, James A. 2007. Eurasian Crossroads: A History of Xinjiang. New York: Columbia University Press.

Millward, James A. 2013. The Silk Road: A Very Short Introduction. New York: Oxford University Press.

Millward, James A. 2018a (2 de febrero). What It's Like to Live in a Surveillance State. The New York Times. https://www.nytimes.com/2018/02/03/ opinion/sunday/china-surveillance-state-uighurs.html.

Millward, James A. 2018b. Old World Geoschemes, Past and Present, and the Belt and Road Initiative as Sino-Silk Roadist Remapping of Afro-Eurasia. Ponencia presentada en Remapping Asian Studies. Granada: 2-23.03.2018.

Perdue, Peter. 1998. Boundaries, Maps and Movement: Chinese, Russian and Mongolian Empires in Early Modern Central Asia. The International History Review 20(2): 253-504. 
Perdue, Peter. 2005. China Marches West: the Qing conquest of Central Eurasia. Cambridge, MA: Harvard University Press.

Pulleyblank, Edwin. G. 2002. Early Contacts between Indo-Europeans and Chinese. En Pulleyblank, Edwin G. (ed.) Central Asia and Non-Chinese Peoples of Ancient China. Burlington: Ashgate, 1-47.

Qarshi, Jamal. 2009. The Conversion to Islam of Satuq Bughra Khan. En Levi, Scott \& Sela, Ron (ed.) Islamic Central Asia: An Anthology of Historical Sources. Bloomington: Indiana University Press, 73-76.

Rawski, Evelyn S. 1996. Presidential Address: Reenvisioning the Qing: The Significance of the Qing Period in Chinese History. The Journal of Asian Studies 55(4): 829-850.

Rawski, Evelyn S. 2015. Early Modern China and Northeast Asia. Cross-Border Perspectives. Cambridge: Cambridge University Press.

Ruiz Gutiérrez, Ana \& Sorroche Cuerva, Miguel Ángel (ed.). 2013. La Ruta de la Seda. Camino de Caminos. Granada: EUG.

Sautman, Barry. 2000. Is Xinjiang an Internal Colony? Inner Asia 2(2): 239271 .

Sherratt, Andrew. 2006. The Trans-Eurasian Exchange: The Prehistory of Chinese Relations with the West. En Mair, Victor (ed.) Contact and Exchange in the Ancient World. Honolulu: University of Hawaii Press, 30-61.

Teggart, Frederik John. 1939. Rome and China: A Study of Correlations in Historical Events. University of California Press.

Thum, Rian. 2014. The Sacred Routes of Uyghur History. Cambridge, MA: Harvard University Press.

Waley-Cohen, Joanna. 2004. The New Qing History. Radical History Review 88: 193-206.

Wu, Guo. 2016. New Qing History: Dispute, Dialog, and Influence. The Chinese Historical Review 23(1): 47-69. 